\title{
AN EXTENSION OF THE LIE BRACKET SOLUTION OF SINGULAR OPTIMAL CONTROLS
}

\author{
Sudhakar Medepalli* and N.X.Vinh ${ }^{\dagger}$ \\ The University of Michigan \\ Ann Arbor, Michigan 48109-2140
}

\begin{abstract}
Singular arcs form a part of the extremal arc in many instances in optimal control theory, the most common one occurring when the control appears linearly in the Hamiltonian. Pontryagin's maximum principle does not yield any information in such cases and we must differentiate the switching function an even number of times with respect to time for the control to appear explicitly. Lie and Poisson Brackets are very elegant and convenient means of expressing this control but in the literature, these solutions have been given only for the case of a single control appearing linearly, which is a case of a totally singular arc. With the help of the concept of the Generalized Hamiltonian, we extend the Poisson Bracket solution to the case of partially singular arcs, where both singular and non-singular controls appear together and these are quite common in the literature. For some special cases, the solution can be expressed in terms of the Lie Brackets also. We illustrate these results with the help of an example of optimal turn to a heading of a spacecraft in atmmpheric flight. We show that the results are identical to those obtained by conventional methods and discuss the advantages of the Lie Bracket method.
\end{abstract}

\section{Introduction}

In optimal control theory, there are many instances where the application of Pontryagin's maximum principle does not yield any information about the control variables. Such cases are termed as the 'Singular Cases' in the literature. The corresponding control is called the singular control and the extremal trajectory, the singular arc. The most common example of these occurs when the control appears linearly in the equations of motion. The singular cases are of interest not only from a theoretical point of view, but also from a practical one. For example, there has been a long debate regarding the op-

*Ph.D.Candidate, Department of Aerospace Engineering, Student Member AIAA.

$\uparrow$ Professor, Department of Aerospace Engineering, Member AIAA.

Copyright (C)American Institute of Aeronautics and A s tronautics, Inc., 1992. All rights reserved. timality of the steady cruise flight of an aircraft which, if at all, must necessarily be a part of a singular $\operatorname{arc}^{1,2,3}$. Singular controls can also occur in various other fields such as chemical process control and economics. See Ref.(4) for an excellent introduction to the subject.

For the case of a single control appearing linearly, necessary conditions have been developed by Kelley et. al ${ }^{5}$ in the late sixties which led to the Kelley-Contensou test and the method of finding the singular control by successive differentiation of the switching function. The results were further extended to multi-input systems by $\mathrm{Goh}^{6,7}$ and Robbins ${ }^{8}$, the latter giving a Generalized Legendre-Clebsch condition. Since then, various authors obtained results on the junction conditions between singular and non-singular arcs and necessary and sufficient conditions for the existence and optimality of singular $\operatorname{arcs}^{4}$. Krener's ${ }^{9}$ higher order maximum principle is a very general theory which includes Pontryagin's principle as a special case.

Concurrent to the above developments, various authors, mostly Russian, have used the elegant and powerful tools of differential geometry, namely the Lie and Poisson Brackets to express the singular control. The survey paper by Gabasov and Kirillova ${ }^{10}$ is an excellent source of a number of these references. As stated in that paper, Poisson's theorem and the Jacobi identity can be used to show that the singular control appears only in an even order derivative of the switching function. More recent articles on these subjects are by Bell", FraserAndrews $^{12}$ and Lamnabhi-Lagerrigue ${ }^{13}$.

In most of the literature listed above, it is assumed that the singular arcs are totally singular (a formal definition will be given later) where all the controls are singular over the entire interval of interest. Especially the Lie and Poisson bracket solutions are given only for singular arcs with only one control variable, with obvious extensions to multi-variable but totally singular cases. Partially singular arcs (a mixture of singular and non-singular controls) occur quite frequently in applications, for example in the flight of a lifting vehicle in 
the atmosphere, where the lift and bank controls can be non-singular while the thrust program is singular.

In what follows, we first give the results for single input case with the control appearing linearly. Definitions and properties of Lie and Poisson Brackets are given. Even though most of these results are quite standard in the literature, we present them in a formal theoremproof format and add our own remarks and observations wherever appropriate. Most of the results and the outlines of the proofs in this section are obtained from the recent paper by Bell ${ }^{11}$. The extensions to the above case are presented by defining partially and totally singular arcs and the concept of Generalized Hamiltonian as used by Robbins ${ }^{8}$. We then verify our results using a standard example in the literature and conclude this paper with a summary.

\section{Previous Work}

In this section we summarize all the results for the case of a scalar control on a singular arc. The statement of the optimal control problem is followed by definitions and properties of the Poisson Brackets. Then the optimal singular control is expressed in terms of the Poisson Brackets. By the simple relation between the Lie and the Poisson Brackets, all the results are expressed in terms of the former.

Let the dynamic system be governed by the following first order ordinary differential equations

$$
\dot{\mathrm{x}}=\mathbf{f}_{\mathbf{0}}(\mathbf{x})+u \mathbf{f}_{\mathbf{1}}(\mathbf{x}),
$$

where, $\mathbf{x}, \mathbf{f}_{\mathbf{0}}, \mathbf{f}_{\mathbf{1}} \in \boldsymbol{R}^{\prime \prime}$ and $\boldsymbol{u} \in \boldsymbol{R}^{\mathbf{1}}, \boldsymbol{x}$ is the state vector and $\boldsymbol{u}$ is the control. Throughout this paper, we assume that all scalar and vector functions are sufficiently smooth in all their arguments so that all their partial derivatives to the required order exist and are continuous. The control $u \in U$, a compact set.

It is desired to obtain the time history of the control $u$ that maximizes the performance index which is a function of the final states (thus the problem is of Mayer type)

$$
\max J=J\left(\mathrm{x}\left(t_{f}\right)\right),
$$

while satisfying certain initial and final conditions

$$
\mathbf{x}\left(t_{0}\right)=\mathbf{x}_{0} \text { and } \Psi\left(\mathbf{x}\left(t_{f}\right)\right)=\mathbf{0},
$$

where $\mathbb{\Psi} \in R^{r}$.

We form the Hamiltonian by introducing the adjoint vector p $E \boldsymbol{R}^{\prime \prime}$ as

$$
\begin{aligned}
H & =\mathbf{p}^{T} \mathbf{f}_{0}+u \mathbf{p}^{T} \mathbf{f}_{1} \\
& =H_{0}(\mathbf{x}, \mathbf{p})+u H_{1}(\mathbf{x}, \mathbf{p}) .
\end{aligned}
$$

Applying the Pontryagin's maximum principle, the adjoint vector satisfies the differential equation

$$
\begin{aligned}
\dot{\mathbf{p}} & =-\left(\frac{\partial H}{\partial \mathbf{x}}\right) \\
& =-\left(\frac{\partial H_{0}}{\partial \mathrm{x}_{0}}\right) r-u\left(\frac{\partial H_{1}}{\partial \mathbf{x}_{\mathbf{f}}}\right) \\
& =-\left(\frac{\partial}{\partial \mathbf{x}}\right) \mathbf{p}-u\left(\frac{\boldsymbol{x}}{\partial \mathbf{x}}\right)
\end{aligned}
$$

and the control is given by

$$
u=\arg \max _{u \in U}(H)
$$

We call $\left(\frac{\partial H}{\partial u}\right)=H_{1}$ the switching function. If $H_{1}=0$ over a non-zero interval of time, the maximum principle gives no information about $u$. In general, whenever $\left(\frac{\partial^{2} H}{\partial u^{2}}\right)=0$, we have a singular case. We must successively differentiate $\boldsymbol{H}_{\mathbf{1}}$ with respect to time until $\boldsymbol{u}$ appears explicitly and it can be shown that this happens, if at all, only in an even order derivative of $H_{1}$. We express these derivatives in terms of Poisson Brackets as shown below.

Definition 1 If $a(\mathbf{x}, \mathbf{p})$ and $b(\mathrm{x}, \mathrm{p})$ are any two scalar functions of $x$ and $\mathrm{p} \in \Re^{n}$, then, the Poisson Bracket of $a$ and $b$ is defined $a s^{14}$

$$
\{a, b\}_{\mathbf{x}, \mathbf{p}}=\left(\frac{\partial b}{\partial \mathbf{x}}\right)^{T}\left(\frac{\partial a}{\partial \mathbf{p}}\right)-\left(\frac{\partial a}{\partial \mathbf{x}}\right)^{T}\left(\frac{\partial b}{\partial \mathbf{p}}\right)
$$

\section{Properties of the Poisson Brackets}

Given three scalar functions $a, b, \mathrm{c}$ of $\boldsymbol{x}$ and $\mathbf{p}$, we have

$$
\begin{aligned}
& \{a, b\}=-\{b, a\} \\
& \{a, 0\}=0 \\
& \{a, a\}=0
\end{aligned}
$$

and the Jacobi identity

$$
\{a,\{b, c\}\}+\{b,\{c, a\}\}+\{c,\{a, b\}\}=0
$$

We need the following lemma before we proceed further.

Lemma 1 If $a(\mathrm{x}, \mathrm{p})$ is a scalar function of $x$ and $\mathrm{p}$, where $x$ and $\mathbf{p}$ are the state and the adjoint variables governed by the differential equations

$$
\dot{\mathrm{x}}=\left(\frac{\partial H}{\partial \mathrm{p}}\right) \text { and } \dot{\mathrm{p}}=-\left(\frac{\partial H}{\partial \mathrm{x}}\right)
$$

then

$$
\frac{d a}{d t}=\left\{H_{0}, a\right\}+u\left\{H_{1}, a\right\}
$$


Proof : Using Eqs.(10), (7) and (4), we get

$$
\begin{aligned}
& \frac{d a}{d t}=\left(\frac{\partial a}{\partial \mathbf{x}}\right)^{T}\left(\frac{d \mathbf{x}}{d t}\right)+\left(\frac{\partial a}{\partial \mathbf{p}}\right)^{T}\left(\frac{d \mathbf{p}}{d t}\right) \\
& =\left(\frac{\partial a}{\partial \mathbf{x}}\right)^{T}\left(\frac{\partial H_{0}}{\partial \mathbf{p}}+u \frac{\partial H_{1}}{\partial \mathbf{p}}\right) \\
& -\left(\frac{\partial a}{\partial \mathbf{p}}\right)^{T}\left(\frac{\partial H_{0}}{\partial \mathbf{x}}+u \frac{\partial H_{1}}{\partial H_{x}}\right) \\
& =\left[\left(\frac{\partial a}{\partial \mathbf{x}}\right)^{T}\left(\frac{\partial H_{0}}{\partial \mathbf{p}}\right)-\left(\frac{\partial H_{0}}{\partial \mathbf{x}}\right)^{T}\left(\frac{\partial a}{\partial \mathbf{p}}\right)\right] \\
& +u\left[\left(\frac{\partial a}{\partial \mathbf{x}}\right)^{T}\left(\frac{\partial H_{1}}{\partial \mathbf{p}}\right)-\left(\frac{\partial H_{1}}{\partial \mathrm{x}}\right)^{T}\left(\frac{\partial a}{\partial \mathbf{p}}\right)\right] \\
& =\left\{H_{0}, a\right\}+u\left\{H_{1}, a\right\}
\end{aligned}
$$

q.e.d.

Note that $u$ appears linearly in the derivative. Following Ref. (9) we define the notation

$$
\begin{aligned}
& d^{i} H_{1}=\left\{H_{0}, d^{i-1} H_{1}\right\}, \\
& d^{0} H_{1}=H_{1}, \quad i=1,2, \ldots
\end{aligned}
$$

With this, we have the following result.

Lemma 2 If, for the optimal control problem given by Eqs. $(1)=(J)$, the singular control appears in the $k$ th time derivative of $H_{1}$, then

$$
\begin{aligned}
\frac{d^{i}}{d t^{i}}\left(\frac{\partial H}{\partial u}\right) & =d^{i} H_{1}=0, \quad i=0,1, \ldots,(k-1) \\
\left\{H_{1}, d^{i-1} H_{1}\right\} & \equiv 0, \quad i=1,2, \ldots,(k-1) \\
\frac{d^{k}}{d t^{k}}\left(\frac{\partial H}{\partial u}\right) & =d^{k} H_{1}+u\left\{H_{1}, d^{k-1} H_{1}\right\}
\end{aligned}
$$

Proof : The first equality is trivial for $i=0$. We prove the first equality by induction. For $i=1$, by Lemma 1 ,

$$
\begin{aligned}
\frac{d}{d t}\left(\frac{\partial H}{\partial u}\right) & =\frac{d H_{1}}{d t} \\
& =\left\{H_{0}, H_{1}\right\}+u\left\{H_{1}, H_{1}\right\}=d^{1} H_{1}
\end{aligned}
$$

Let the first equality be true for $\mathbf{j}<k-1$, i.e.,

$$
\frac{d^{j}}{d t^{j}}\left(\frac{\partial H}{\partial u}\right)=d^{j} H_{1}
$$

Then, using Lemma 1,

$$
\begin{aligned}
\frac{d^{j+1}}{d t^{j+1}}\left(\frac{\partial H}{\partial u}\right) & =\frac{d}{d t}\left(d^{j} H_{1}\right) \\
& =\left\{H_{0}, d^{j} H_{1}\right\}+u\left\{H_{1}, d^{j} H_{1}\right\}(14) \\
& =d^{j+1} H_{1}+u\left\{H_{1}, d^{j} H_{1}\right\}
\end{aligned}
$$

But since the singular control appears for the first time in the $k$ th derivative, we have,

$$
\bar{\partial}\left[\frac{d^{i}}{\partial u}\left(\frac{\partial H}{d t^{i}}\right)\right] \equiv 0, \quad i=0,1, \ldots,(k-1)
$$

Thus, since $(j+1)<k$,

$$
\frac{\partial}{\partial u}\left[\frac{d^{j+1}}{d t^{j+1}}\left(\frac{\partial H}{\partial u}\right)\right]=\left\{H_{1}, d^{j} H_{1}\right\} \equiv 0
$$

and

$$
\frac{d^{j+1}}{d t^{j+1}}\left(\frac{\partial H}{\partial u}\right)=d^{j+1} H_{1}
$$

Thus we proved the first equality for $i=0,1, \ldots(E-1)$. At the same time, we also see from Eqs. (15) and (16), that the second equality is true for $i=1,2, \ldots(k-1)$.

Finally, to prove the last result, using $j=(k-1)$ in Eq.(14), we have

$$
\frac{d^{k}}{d t^{k}}\left(\frac{\partial H}{\partial u}\right)=d^{k} H_{1}+u\left\{H_{1}, d^{k-1} H_{1}\right\}
$$

q.e.d.

Lemma 3 Given $\left\{H_{1}, d^{i} H_{1}\right\} \mathbf{E} 0, i=0,1, \ldots,(k-2)$, then, $V i=0,1, \ldots,(I-1)$ and $\forall l=1,2, \ldots,(E-1)$,

$$
\left\{d^{i} H_{1}, d^{l-i} H_{1}\right\}=-\left\{d^{(i+1)} H_{1}, d^{l-(i+1)} H_{1}\right\}
$$

Proof : We prove this by principle of complete induction $^{16}$. For $\boldsymbol{i}=0$, we have using the Jacobi identity,

$$
\begin{aligned}
&\left\{H_{1}, d^{l} H_{1}\right\} \\
&=\quad\left\{H_{1},\left\{H_{0}, d^{l-1} H_{1}\right\}\right\} \quad(\text { def. }) \\
&=-\left\{H_{0},\left\{d^{l-1} H_{1}, H_{1}\right\}\right\} \\
&-\left\{d^{l-1} H_{1},\left\{H_{1}, H_{0}\right\}\right\} \quad(\text { Jucobi }) \\
&=-\left\{\left\{H_{0}, H_{1}\right\}, d^{l-1} H_{1}\right\}\left(\left\{H_{1}, d^{l-1} H_{1}\right\} \equiv 0\right) \\
&=-\left\{d^{1} H_{1}, d^{l-1} H_{1}\right\} \quad \text { (def.) }
\end{aligned}
$$

Let Eq.(19) be true for $i=0,1, \ldots,(\mathbf{j}-1), j<l$. Then we need to show that

$$
\left\{d^{j} H_{1}, d^{l-j} H_{1}\right\}=-\left\{d^{j+1)} H_{1}, d^{l-(j+1)} H^{1}\right\}
$$

$$
\begin{aligned}
\text { LHS } & \\
= & \left\{d^{j} H_{1},\left\{H_{0}, d^{l-(j+1)} H_{1}\right\} \quad(\text { def. })\right. \\
= & -\left\{H_{0},\left\{d^{l-(j+1)} H_{1}, d^{j} H_{1}\right\}\right\} \\
& -\left\{d^{l-(j+1)} H_{1},\left\{d^{j} H_{1}, H_{0}\right\}\right\} \quad(\text { Jacobi }) \\
= & -\left\{H_{0},\left\{d^{(l-1)-j} H_{1}, d^{j} H_{1}\right\}\right\} \\
& -\left\{d^{j+1} H_{1}, d^{l-(j+1)} H_{1}\right\}
\end{aligned}
$$


Let us consider the first term on the right hand side. Applying Eq. (19) backwards with $(I-1)$ instead of $l$, we get

$$
\begin{aligned}
& -\left\{d^{(l-1)-j} H_{1}, d^{j} H_{1}\right\} \\
& =\left\{d^{j} H_{1}, d^{(1-1)-j} H_{1}\right\} \quad(8) \\
& =-\left\{d^{j-1} H_{1}, d^{(l-1)-(j-1)} H_{1}\right\} \quad((19), i=(j-1)) \\
& =+\left\{d^{j-2} H_{1}, d^{(l-1)-(j-2)} H_{1}\right\} \quad((19), i=(j-2)) \\
& =\vdots \\
& =(-1)^{j+1}\left\{d^{j-j} H_{1}, d^{(l-1)-(j-j)} H_{1}\right\} \\
& =(-1)^{j+1}\left\{H_{1}, d^{(l-1)} H_{1}\right\} \\
& \equiv 0 \quad \text { (hyp., } l<k)
\end{aligned}
$$

q.e.d.

Corollary 1 For the same Hypothesis as in Lemma 3, we have

$$
\begin{array}{r}
\left\{d^{i} H_{1}, d^{l-i} H_{1}\right\}=-(1)^{i+1}\left\{H_{1}, d^{l} H_{1}\right\} \\
V i=0,1, \ldots,(I-1) \text { and } \forall l=1,2, \ldots,(k-1) .
\end{array}
$$

Proof : Apply Lemma 3 backwards $\boldsymbol{i}$ times as in Eq. (21).

Corollary $\mathbf{2}$ For the same Hypothesis as in Lemma 3,

$$
\left\{d^{i} H_{1}, d^{l-i} H_{1}\right\} \equiv 0
$$

$V i=0,1, \ldots,(I-1)$ and $\forall l=1,2, \ldots,(k-2$.

Proof: Corollary 1 and hypothesis of Lemma 3.

Corollary 3 For the same Hypothesis as in Lemma 3,

$$
\left\{d^{i} H_{1}, d^{(k-1)-i} H_{1}\right\}=(-1)^{i+1}\left\{H_{1}, d^{(k-1)} H_{1}\right\}
$$

Proof : Use Corollary $l$ with $l=(k-1)$.

Theorem 1 If, for the problem stated in (1) = (3) $u$ appears in the $k$ th derivative of $H_{1}$ (linearly) on a singular arc, then $k$ is even.

Proof : We prove that if $k$ is odd, then the coefficient of $u$ in the $k$ th derivative is identically zero. Let $k=$ $(2 q+1)$, where $q \geq 0$. Then from Lemma 2 , we have

$$
\begin{aligned}
& \left\{H_{1}, d^{i} H_{1}\right\} \equiv 0, i=0,1, \ldots,(2 q-1) \\
& \frac{d^{k}}{d t^{k}}\left(\frac{\partial H}{\partial u}\right)=d^{2 q+1} H_{1}+u\left\{H_{1}, d^{2 q} H_{1}\right\}
\end{aligned}
$$

Using Corollary 3, with $\mathrm{k}=(2 q+1)$ and $i=q$ and $\mathrm{Eq}$. (8),

$$
(-1)^{q+1}\left\{H_{1}, d^{2 q} H_{1}\right\}=\left\{d^{q} H_{1}, d^{q} H_{1}\right\} \equiv 0
$$

Definition 2 If $u$ appears explicitly in the $2 q$ th derivative of the switching function, then, $q$ is called the order of the singular arc.

Theorem 2 The Generalized Legendre-Clebsch necessary condition for the optimality of the singular arc is given by

$$
\begin{aligned}
& (-1)^{q+1} \frac{q}{\vec{d} u}\left[\frac{d^{2 q}}{d t^{2 q}}(\underset{+}{\mid+})\right] \\
& =(-1)^{q+1}\left\{H_{1}, d^{2 q-1} H_{1}\right\} \geq 0
\end{aligned}
$$

Proof : See Ref. (5).

We can express all the above results in terms of Lie Brackets which are defined as given below.

Definition 3 Let $\mathbf{f}$ and $\boldsymbol{g} \in R^{n}$ be vector functions of $\mathbf{x}$. Then the Lie Bracket of $\mathbf{f}$ and $\mathbf{g}$ is defined as:

$$
(\mathbf{f}, \mathbf{g})=\left(\begin{array}{c}
\partial g \\
\partial \mathbf{x}
\end{array}\right) \mathbf{f}-\left(\begin{array}{l}
\partial \mathbf{f} \\
\partial x \\
\partial \mathbf{x}
\end{array}\right) \mathbf{g}
$$

where, $\left(\frac{\partial \mathbf{f}}{\partial \mathbf{x}}\right)$ and $\left(\frac{\partial \mathrm{g}}{\partial \mathrm{x}}\right)$ are the $(n \times n)$ Jacobian matrices.

Relation between Lie and Poisson Brackets ${ }^{11}$

Lemma 4 Given the vector functions $\mathbf{f}, \mathbf{g}$ of $\mathrm{x}$ and $\mathbf{p}$, the latter being governed by the (IO), we have:

$$
\left\{\mathbf{p}^{T} \mathbf{f}, \mathbf{p}^{T} \mathbf{g}\right\}=\mathbf{p}^{T}(\mathbf{f}, \mathbf{g})
$$

\section{Proof:}

$$
\begin{aligned}
\left\{\mathbf{p}^{T} \mathbf{f}, \mathbf{p}^{T} \mathbf{g}\right\}= & {\left[\frac{\partial}{\partial \mathbf{x}}\left(\mathbf{p}^{T} \mathbf{g}\right)\right]^{T}\left[\frac{\partial}{\partial \mathbf{p}}\left(\mathbf{p}^{T} \mathbf{f}\right)\right] } \\
& -\left[\frac{\partial}{\partial \mathbf{x}}\left(\mathbf{p}^{T} \mathbf{f}\right)\right]^{T}\left[\frac{\partial}{\partial \mathbf{p}}\left(\mathbf{p}^{T} \mathbf{g}\right)\right] \\
= & \mathbf{p}^{T}\left(\frac{\partial \mathbf{g}}{\partial \mathbf{x}}\right) \mathbf{f}-\mathbf{p}^{T}\left(\frac{\partial \mathbf{f}}{\partial \mathbf{x}}\right) \mathbf{g} \\
= & \mathbf{p}^{T}(\mathbf{f}, \mathbf{g})
\end{aligned}
$$

q.e.d.

The following properties of Lie Brackets can be easily verified from the corresponding ones for the Poisson Brackets.

\section{Properties of Lie Brackets}

Given three vector functions $\mathbf{f}, \mathbf{g}, \mathbf{h}$ of $\mathbf{x}$ and the null vector 8 , we have ${ }^{11}$,

$$
\begin{aligned}
& (\mathbf{g}, \mathbf{f})=-(\mathbf{f}, \mathbf{g}) \\
& (\mathbf{f}, \theta)=\theta \\
& (\mathbf{f}, \mathbf{f})=\theta
\end{aligned}
$$


and the Jacobi Identity

$$
(\mathfrak{f},(\mathbf{g}, \mathbf{h}))+(\mathbf{g},(\mathbf{h}, \mathbf{f}))+(\mathbf{h},(\mathbf{f}, \mathbf{g}))=\theta
$$

If $\mathbf{x}, \mathbf{p}$ are governed by the canonical equations (10), then,

$$
\frac{d}{d t}\left(\mathbf{p}^{T} \mathbf{g}\right)=\mathbf{p}^{T}\left(\mathbf{f}_{0}, \mathbf{g}\right)+u \mathbf{p}^{T}\left(\mathbf{f}_{1}, \mathbf{g}\right)
$$

All the results of the previous lemmas, theorem and corollaries can be translated in terms of the Lie Brackets using Eq.(28)

Remark 1 For a singular arc of order one, the 2nd derivative of the switching function has the following form for the problem defined by Eqs. (1) - (\$).

$$
\begin{aligned}
& \frac{d^{2}}{d t^{2}}\left(\frac{\partial H}{\partial u}\right) \\
& \quad=\left\{H_{0},\left\{H_{0}, H_{1}\right\}\right\}+u\left\{H_{1},\left\{H_{0}, H_{1}\right\}\right\} \\
& =\mathbf{p}^{T}\left(\mathbf{f}_{\mathbf{0}},\left(\mathbf{f}_{\mathbf{0}}, \mathbf{f}_{\mathbf{1}}\right)\right)+u \mathbf{p}^{T}\left(\mathbf{f}_{\mathbf{1}},\left(\mathbf{f}_{\mathbf{0}}, \mathbf{f}_{\mathbf{1}}\right)\right)
\end{aligned}
$$

Remark 2 Extension of the above results to the case of multiple inputs appearing linearly, where the arc is still totally singular, is quite straight forward.

\section{Extension of the Results}

Now we try to extend the above results to the case of partially singular arcs. First we state the problem and give some definitions, introducing the important concept of the Generalized Hamiltonian.

Given a system whose states are governed by a set of first order ordinary differential equations,

$$
\dot{\mathbf{x}}=\mathbf{f}(\mathbf{x}, \mathbf{w}),
$$

where $\mathbf{x} \in R^{\prime \prime}$ is the state vector w $\mathrm{E} \Omega \mathrm{C} R^{m+1}$ is the control vector and $\Omega$ is a compact set, it is desired to maximize the performance index

$$
\max \quad J=J\left(x\left(t_{j}\right)\right),
$$

of the final states subject to the end conditions,

$$
\mathbf{x}\left(t_{0}\right)=\mathbf{x}_{0} \text { and } \mathbf{\Psi}\left(\mathbf{x}\left(t_{f}\right)\right)=0
$$

Thus, once again, the problem is of Mayer type. Introducing the adjoint vector $\mathrm{p}$, we form the Hamiltonian

$$
H=g^{\boldsymbol{T}_{\mathbf{f}}}
$$

By the Pontryagin's maximum principle, the first order necessary conditions for an interior control are

$$
\left(\frac{\partial H}{\partial w}\right)=0
$$

and the state and the adjoint vectors satisfy the canonical system given by (10) The second order necessary conditions are that the $(m+1) \mathbf{x}(m+1)$ Jacobian matrix

$$
\left(\frac{\partial^{2} H}{\partial w^{2}}\right) \leq 0
$$

Definition 4 The extremal trajectory is called totally singular ${ }^{4,13}$ on $\left[t_{0}, t_{f}\right]$ if and only if

$$
\left(\frac{\partial^{2} H}{\partial \mathrm{w}^{2}}\right)=0 \quad v t \mathrm{E}\left[t_{0}, t_{j}\right]
$$

Definition 5 The extremal trajectory is called partially singular ${ }^{4,13}$ on $\left[t_{0}, t_{f}\right]$ if and only if

$$
\begin{gathered}
\operatorname{det}\left(\frac{\partial^{2} H}{\partial \mathbf{w}^{2}}\right)=0 \quad v t E\left[t_{0}, t_{f}\right] \\
\text { and } \\
\left(\frac{\partial^{2} H}{\partial \mathbf{w}^{2}}\right) \quad \neq \quad 0
\end{gathered}
$$

\section{A Special Case}

For the sake of simplicity let us assume that the equations of motion are of the form

$$
\dot{\mathbf{x}}=\mathbf{f}\left(\mathbf{x}_{,} \mathbf{v}_{\mathbf{0}}\right)+u \mathbf{g}\left(\mathbf{x}, \mathbf{v}_{\mathbf{1}}\right)
$$

where $\mathrm{x}, \mathfrak{\mathbb { L }}, \mathrm{g} \in R^{\mathrm{n}}, \mathrm{v}_{\mathbf{0}} \in R^{m_{0}}, \mathrm{v}_{\mathbf{1}} \in R^{m_{1}}$ and $\mathrm{u} \in R^{1}$. This form occurs quite frequently, for example, in the flight of a lifting vehicle in atmosphere where $v_{0}$ represents the lift and the bank controls, $\mathbf{v}_{\mathbf{1}}$ represents the thrust direction and $u$ represents the thrust magnitude.

The Hamiltonian is given by

$$
\begin{aligned}
H & =\mathbf{p}^{\boldsymbol{T}} \mathbf{f}+u \mathbf{p}^{\boldsymbol{T}} \mathbf{g} \\
& =H_{0}\left(\mathbf{x}, \mathbf{p}, \mathbf{v}_{\mathbf{0}}\right)+u H_{1}\left(\mathbf{x}, \mathbf{p}, \mathbf{v}_{\mathbf{1}}\right)
\end{aligned}
$$

Let $\mathbf{v}_{\mathbf{0}}$ and $\mathbf{v}_{\mathbf{1}}$ be interior optimal controls. Thus,

$$
\begin{aligned}
& \left(\frac{\partial H_{0}}{\partial \mathbf{v}_{\mathbf{0}}}\right)=0, \mathbf{D}_{\mathbf{0}}=\left(\frac{\partial^{2} H_{0}}{\partial \mathbf{v}_{\mathbf{0}}{ }^{2}}\right)<0 \\
& \left(\frac{\partial H_{1}}{\partial \mathbf{v}_{\mathbf{1}}}\right)=0, \mathbf{D}_{\mathbf{1}}=\left(\frac{\partial^{2} H_{1}}{\partial \mathbf{v}_{\mathbf{1}}{ }^{2}}\right)<0
\end{aligned}
$$

Writing

$$
\mathbf{w}=\left[\begin{array}{c}
u \\
\mathbf{v}_{\mathbf{0}} \\
\mathbf{v}_{\mathbf{1}}
\end{array}\right] \text {, }
$$


we get

$$
\left(\frac{\partial^{2} H}{\partial \mathbf{w}^{2}}\right)=\left[\begin{array}{ccc}
0 & \theta & \theta \\
\theta & \mathbf{D}_{0} & \Theta \\
\theta & \Theta & \mathbf{D}_{1}
\end{array}\right]
$$

where $\theta$ is the null vector and $\Theta$ is the null matrix of appropriate dimensions. Clearly the Hessian matrix has a rank deficiency of $\mathbf{1}$ and this is a case of a partially singular arc.

Since we assumed that $\mathbf{D}_{\mathbf{0}}$ and $\mathbf{D}_{\mathbf{1}}$ are non-singular, we can express $\mathbf{v}_{\mathbf{0}}$ and $\mathbf{v}_{\mathbf{1}}$ as functions of $x a n d p$ using the first order necessary conditions given in (43) and (44) and the implicit functions theorem.

Definition 6 We define the Generalized Variational Hamiltonian or simply the Generalized Hamiltonian ${ }^{8}$ as

$$
\mathcal{H}(\mathbf{x}, \mathbf{p}, u)=H\left(\mathbf{x}, \mathbf{p}, \mathbf{v}_{\mathbf{0}}(\mathbf{x}, \mathbf{p}), \mathbf{v}_{\mathbf{1}}(\mathbf{x}, \mathbf{p}), u\right)
$$

Note that the Generalized Hamiltonian is no longer linear in the adjoint variables.

Lemma 5 The canonical equations generated by $\mathbf{H}$ and $\mathcal{H}$ are identical. In other words,

$$
\left(\frac{\partial H}{\partial \mathbf{x}}\right)=\left(\frac{\partial \mathcal{H}}{\partial \mathbf{x}}\right) \text { and }\left(\frac{\partial H}{\partial \mathbf{p}}\right)=\left(\frac{\partial \mathcal{H}}{\partial \mathbf{p}}\right)
$$

Proof: Using Eq.(47) and the first order necessary conditions in (43) and (44), we get

$$
\begin{aligned}
\left(\frac{\partial \mathcal{H}}{\partial \mathbf{x}}\right)= & \left(\frac{\partial H}{\partial \mathbf{x}}\right)+\left(\frac{\partial \mathbf{v}_{0}}{\partial \mathbf{x}}\right)^{T}\left(\frac{\partial H}{\partial \mathbf{v}_{\mathbf{0}}}\right) \\
& +\left(\frac{\partial \mathbf{v}_{1}}{\partial \mathbf{x}}\right)^{T}\left(\frac{\partial H}{\partial \mathbf{v}_{1}}\right)=\left(\frac{\partial H}{\partial \mathbf{x}}\right) \\
\left(\frac{\partial \mathcal{H}}{\partial \mathbf{p}}\right)= & \left(\frac{\partial H}{\partial \mathbf{p}}\right)+\left(\frac{\partial \mathbf{v}_{\mathbf{0}}}{\partial \mathbf{p}}\right)^{T}\left(\frac{\partial H}{\partial \mathbf{v}_{\mathbf{0}}}\right) \\
& +\left(\frac{\partial \mathbf{v}_{1}}{\partial \mathbf{p}}\right)^{T}\left(\frac{\partial H}{\partial \mathbf{v}_{1}}\right)=\left(\frac{\partial H}{\partial \mathbf{p}}\right)
\end{aligned}
$$

q.e.d.

Clearly, we can write the Generalized Hamiltonian in the form,

$$
\begin{aligned}
\mathcal{H} & =\mathcal{H}_{0}(\mathbf{x}, \mathbf{p})+u \mathcal{H}_{1}(\mathbf{x}, \mathbf{p}) \\
& =H_{0}\left(\mathbf{x}, \mathbf{p}, \mathbf{v}_{\mathbf{0}}(\mathbf{x}, \mathbf{p})\right)+u H_{1}\left(\mathbf{x}, \mathbf{p}, \mathbf{v}_{\mathbf{1}}(\mathbf{x}, \mathbf{p})\right)
\end{aligned}
$$

i.e., $\boldsymbol{u}$ appears linearly. The state and the adjoint vectors satisfy the canonical system,

$$
\dot{\mathrm{x}}=\left(\frac{\partial \mathcal{H}}{\partial \mathbf{p}}\right) \text { and } \dot{\mathrm{p}}=-\left(\frac{\partial \mathcal{H}}{\partial \mathbf{x}}\right)
$$

Remark 3 Theform of the above two equations is identical to that of the single input case considered earlier. Thus all the results for the singular control $\mathbf{u}$ are equally well applicable here with $\mathcal{H}_{0}$ and $\mathcal{H}_{1}$ taking the place of $H_{0}$ and $H_{1}$ in the Poisson Brackets.
Remark 4 Since $\mathcal{H}_{0}$ and $\mathcal{H}_{1}$ are not linear in $\mathrm{p}$, we cannot, in general, express $\mathbf{u}$ in terms of Lie Brackets except in the special case we considered in (40).

Lemma 6 For the problem defined in (40),

$$
\frac{d \mathbf{v}_{0}}{d t}=-\mathbf{D}_{\mathbf{0}}^{-1}\left[\left\{H_{0},\left(\frac{\partial H_{0}}{\partial \mathbf{v}_{0}}\right)\right\}+u\left\{H_{1},\left(\frac{\partial H_{0}}{\partial \mathbf{v}_{0}}\right)\right\}\right]
$$

where we define $\left\{a,\left(\frac{\partial H_{0}}{\partial v_{0}}\right)\right\}$ as a $\left(m_{0} \times 1\right)$ column vector such that

$$
\left\{a,\left(\frac{\partial H_{0}}{\partial \mathbf{v}_{0}}\right)\right\}_{i}=\left\{a,\left(\frac{\partial H_{0}}{\partial v_{0_{i}}}\right)\right\}
$$

Proof : The implicit equation $\left(\frac{\partial \boldsymbol{H}_{0}}{\partial \mathbf{v}_{0}}\right)=0$ expresses $\mathbf{v}_{0}$ $\boldsymbol{s}$ a function of $\mathbf{x}$ and $\mathbf{p}$. Differentiating by chain rule we get

$$
\frac{d \mathrm{y}_{0}}{d t}=\left(\frac{\partial \mathrm{v}_{0}}{\partial \mathrm{x}}\right)\left(\frac{d \mathrm{x}}{d t}\right)+\left(\frac{\partial \mathrm{v}_{0}}{\partial \mathrm{p}}\right)\left(\frac{d \mathrm{p}}{d t}\right)
$$

Since we assumed that the Jacobian matrix $\mathbf{D}_{\mathbf{0}}$ is non-singular, we can evaluate the partials $\left(\frac{\partial \mathbf{v}_{\mathbf{0}}}{\partial \mathrm{X}}\right)$ and $\left(\frac{\partial \mathbf{v}_{\mathbf{0}}}{\partial \mathbf{p}}\right)$ using the implicit functions theorem (see Appendix).

$$
\begin{aligned}
& \left(\frac{\partial \mathbf{v}_{\mathbf{0}}}{\partial \mathbf{x}}\right)=-\mathbb{D}_{0}^{-1}\left[\frac{\partial}{\partial \mathbf{x}}\left(\frac{\partial H_{0}}{\partial \mathbf{v}_{0}}\right)\right] \\
& \left(\frac{\partial \mathbf{v}_{\mathbf{0}}}{\partial \mathbf{p}}\right)=-\mathbf{D}_{\mathbf{0}}^{-1}\left[\frac{\partial}{\partial \mathbf{p}}\left(\frac{\partial H_{0}}{\partial \mathbf{v}_{0}}\right)\right]
\end{aligned}
$$

Combining Eqs. (52) and (53), and using the canonical equations along with Eq. (41), we have

$$
\begin{array}{rr}
\frac{d \mathbf{v}_{\mathbf{0}}}{d t} & -\mathbf{D}_{\mathbf{0}}{ }^{-1}\left[\frac{\partial}{\partial \mathbf{x}}\left(\frac{\partial H_{0}}{\partial \mathbf{v}_{\mathbf{0}}}\right)\right]\left[\left(\frac{\partial H_{0}}{\partial \mathbf{p}}\right)+u\left(\frac{\partial H_{1}}{\partial \mathbf{p}}\right)\right] \\
& +\mathbf{D}_{0}{ }^{-1}\left[\frac{\partial}{\partial \mathbf{p}}\left(\frac{\partial H_{0}}{\partial \mathbf{v}_{0}}\right)\right]\left[\left(\frac{\partial H_{0}}{\partial \mathbf{x}}\right)+u\left(\frac{\partial H_{1}}{\partial \mathbf{x}}\right)\right] \\
= & -\mathbf{D}_{\mathbf{0}}{ }^{-1}\left[\left(\frac{\partial}{\partial \mathbf{x}}\left(\frac{\partial H_{0}}{\partial \mathbf{v}_{0}}\right)\right)\left(\frac{\partial H_{0}}{\partial \mathbf{p}}\right)\right. \\
& \left.-\left(\frac{\partial}{\partial \mathbf{p}}\left(\frac{\partial H_{0}}{\partial \mathbf{v}_{0}}\right)\right)\left(\frac{\partial H_{0}}{\partial \mathbf{x}}\right)\right] \\
& -u \mathbf{D}_{\mathbf{0}}{ }^{-1}\left[\left(\frac{\partial}{\partial \mathbf{x}}\left(\frac{\partial H_{0}}{\partial \mathbf{v}_{0}}\right)\right)\left(\frac{\partial H_{1}}{\partial \mathbf{p}}\right)\right. \\
- & \left.-\left(\frac{\partial}{\partial \mathbf{p}}\left(\frac{\partial H_{0}}{\partial \mathbf{v}_{0}}\right)\right)\left(\frac{\partial H_{1}}{\partial \mathbf{x}}\right)\right] \\
= & -\mathbf{D}_{\mathbf{0}}{ }^{-1}\left[\left\{H_{0},\left(\frac{\partial H_{0}}{\partial \mathbf{v}_{\mathbf{0}}}\right)\right\}+u\left\{H_{1},\left(\frac{\partial H_{0}}{\partial \mathbf{v}_{\mathbf{0}}}\right)\right\}\right]
\end{array}
$$

q.e.d.

In a similar fashion we have 
Lemma 7 For the problem defined in (40),

$$
\frac{d \mathbf{v}_{1}}{d t}=-\mathbf{D}_{1}^{-1}\left[\left\{H_{0},\left(\frac{\partial H_{1}}{\partial \mathbf{v}_{\mathbf{1}}}\right)\right\}+u\left\{H_{1},\left(\frac{\partial H_{1}}{\partial \mathbf{v}_{\mathbf{1}}}\right)\right\}\right]
$$

With these results, we can prove the following result

Lemma 8 If $\boldsymbol{a}$ is any scalar function of $\mathrm{x}, \mathrm{p}, \mathrm{v}_{0}, \mathrm{v}_{\mathbf{1}}$, the latter being defined in (40), then,

$$
\begin{aligned}
& \frac{d a}{d t} \\
&=\left\{H_{0}, a\right\}+u\left\{H_{1}, a\right\} \\
&-\left(\frac{\partial a}{\partial \mathbf{v}_{0}}\right)^{T} \mathbf{D}_{\mathbf{0}}{ }^{-1}\left[\left\{H_{0},\left(\frac{\partial H_{0}}{\partial \mathbf{v}_{\mathbf{0}}}\right)\right\}\right. \\
&\left.+u\left\{H_{1},\left(\frac{\partial H_{0}}{\partial \mathbf{v}_{\mathbf{0}}}\right)\right\}\right] \\
&-\left(\frac{\partial a}{\partial \mathbf{v}_{\mathbf{1}}}\right)^{T} \mathbf{D}_{\mathbf{1}}{ }^{-1}\left[\left\{H_{0},\left(\frac{\partial H_{1}}{\partial \mathbf{v}_{\mathbf{1}}}\right)\right\}\right. \\
&\left.+u\left\{H_{1},\left(\frac{\partial H_{1}}{\partial \mathbf{v}_{1}}\right)\right\}\right]
\end{aligned}
$$

Proof: Note that

$$
\begin{aligned}
d a & \left(\frac{\partial a}{\partial \mathrm{x}}\right)^{T}\left(\frac{d \mathbf{x}}{d t}\right)+\left(\frac{\partial a}{\partial \mathrm{p}}\right)^{T}\left(\frac{d \mathrm{p}}{d t}\right) \\
& +\left(\frac{\partial a}{\partial \mathbf{v}_{0}}\right)^{T}\left(\frac{d \mathbf{v}_{0}}{d t}\right)+\left(\frac{\partial a}{\partial \mathbf{v}_{1}}\right)^{T}\left(\frac{d \mathbf{v}_{1}}{d t}\right)
\end{aligned}
$$

Similar to Lemma 1 , the first two terms on the right hand side in the above equation lead to the first two Poisson Brackets on the right hand side of Eq. (54). The next two terms result from Lemmas 6 and 7. q.e.d.

We need two more results before we prove the final theorem.

Lemma 9 For the problem defined in (40),

$$
\frac{\partial}{\partial \mathbf{v}_{0}}\left\{H_{0}, H_{1}\right\}=-\left\{H_{1},\left(\frac{\partial H_{0}}{\partial \mathbf{v}_{0}}\right)\right\}
$$

Proof : Due to the fact that $H_{0}$ and $H_{1}$ are not functions of $\mathbf{v}_{\mathbf{1}}$ and $\mathbf{v}_{0}$ respectively, we have

$$
\left(\frac{\partial H_{0}}{\partial \mathbf{v}_{1}}\right)=0, \text { and }\left(\begin{array}{l}
\partial H_{*} \\
\partial H_{1} \\
\partial \mathbf{v}_{\mathbf{0}}
\end{array}\right)=0
$$

Using the above results along with the first order necessary conditions given in (43) and (44), we get

$$
\frac{\partial}{\partial \mathbf{v}_{0}}\left\{H_{0}, H_{1}\right\}
$$

$$
\begin{aligned}
= & \frac{\partial}{\partial \mathbf{v}_{0}}\left[\left(\frac{\partial H_{1}}{\partial \mathbf{x}}\right)^{T}\left(\frac{\partial H_{0}}{\partial \mathbf{p}}\right)-\left(\frac{\partial H_{0}}{\partial \mathbf{x}}\right)^{T}\left(\frac{\partial H_{1}}{\partial \mathbf{p}}\right)\right] \\
= & {\left[\frac{\partial}{\partial \mathbf{v}_{\mathbf{0}}}\left(\frac{\partial H_{0}}{\partial \mathbf{p}}\right)\right]^{T}\left(\frac{\partial H_{1}}{\partial \mathbf{x}}\right) } \\
& -\left[\frac{\partial}{\partial \mathbf{v}_{0}}\left(\frac{\partial H_{0}}{\partial \mathbf{x}}\right)\right]^{T}\left(\frac{\partial H_{1}}{\partial \mathbf{p}}\right) \\
= & {\left[\frac{\partial}{\partial \mathbf{p}}\left(\frac{\partial H_{0}}{\partial \mathbf{v}_{0}}\right)\right]\left(\frac{\partial H_{1}}{\partial \mathbf{x}}\right)-\left[\frac{\partial}{\partial \mathbf{x}}\left(\frac{\partial H_{0}}{\partial \mathbf{v}_{0}}\right)\right]\left(\frac{\partial H_{1}}{\partial \mathbf{p}}\right) } \\
= & -\left\{H_{1},\left(\frac{\partial H_{0}}{\partial \mathbf{v}_{0}}\right)\right\}
\end{aligned}
$$

q.e.d.

Similarly we can show that

Lemma 10 For the problem defined in (40),

$$
\frac{\partial}{\partial \mathbf{v}_{\mathbf{1}}}\left\{H_{0}, H_{1}\right\}=\left\{H_{0},\left(\frac{\partial H_{1}}{\partial \mathbf{v}_{\mathbf{1}}}\right)\right\}
$$

Combining all the above results, we have

Theorem 3 If $u$ is a singular optimal control of order one for the problem described by Eq. (40), then $u$ is given by

$$
\mathcal{A}+u \mathcal{B}=0
$$

where,

$$
\begin{aligned}
\mathcal{A}= & \left\{H_{0},\left\{H_{0}, H_{1}\right\}\right\} \\
& +\left\{H_{1},\left(\frac{\partial H_{0}}{\partial \mathbf{v}_{0}}\right)\right\}^{T}\left(\frac{\partial^{2} H_{0}}{\partial \mathbf{v}_{0}^{2}}\right)^{-1}\left\{H_{0},\left(\frac{\partial H_{0}}{\partial \mathbf{v}_{0}}\right)\right\} \\
& -\left\{H_{0},\left(\frac{\partial H_{1}}{\partial \mathbf{v}_{1}}\right)\right\}^{T}\left(\frac{\partial^{2} H_{1}}{\partial \mathbf{v}_{1}^{2}}\right)^{-1}\left\{H_{0},\left(\frac{\partial H_{1}}{\partial \mathbf{v}_{1}}\right)\right\}
\end{aligned}
$$

and

$$
\begin{aligned}
\mathcal{B}= & \left\{H_{1},\left\{H_{0}, H_{1}\right\}\right\} \\
& +\left\{H_{1},\left(\frac{\partial H_{0}}{\partial \mathbf{v}_{\mathbf{0}}}\right)\right\}^{T}\left(\frac{\partial^{2} H_{0}}{\partial \mathbf{v}_{\mathbf{0}}^{2}}\right)^{-1}\left\{H_{1},\left(\frac{\partial H_{0}}{\partial \mathbf{v}_{\mathbf{0}}}\right)\right\} \\
& -\left\{H_{0},\left(\frac{\partial H_{1}}{\partial \mathbf{v}_{\mathbf{1}}}\right)\right\}^{T}\left(\frac{\partial^{2} H_{1}}{\partial \mathbf{v}_{\mathbf{1}}^{2}}\right)^{-1}\left\{H_{1},\left(\frac{\partial H_{1}}{\partial \mathbf{v}_{\mathbf{1}}}\right)\right\}
\end{aligned}
$$

The Legendre-Clebsch condition implies that $\mathfrak{B}>0$.

Proof : To obtain $u$, we differentiate $\left(\frac{\partial H}{\partial u}\right)=H_{1}$ twice (since the singular arc is of degree one). Using Lemma 8 with $\boldsymbol{a}=H_{1}$ for the first derivative and using (43), (44) and (56), we get

$$
\frac{d H_{1}}{d t}-\left\{H_{0}, H i\right)
$$


To obtain the second derivative, we use Lemma 8 again but this time with $a=\left\{H_{0}, H_{1}\right\}$ and use Lemmas 9 and 10 to get

$$
\begin{aligned}
\frac{d^{2}}{d t^{2}}\left(\frac{\partial H}{\partial u}\right) & \\
= & \frac{d}{d t}\left\{H_{0}, H_{1}\right\} \\
= & \left\{H_{0},\left\{H_{0}, H_{1}\right\}\right\}+u\left\{H_{1},\left\{H_{0}, H_{1}\right\}\right\} \\
& +\left\{H_{1},\left(\frac{\partial H_{0}}{\partial \mathbf{v}_{\mathbf{0}}}\right)\right\}^{T} \mathbf{D}_{\mathbf{0}}^{-1}\left[\left\{H_{0},\left(\frac{\partial H_{0}}{\partial \mathbf{v}_{\mathbf{0}}}\right)\right\}\right. \\
& \left.+u\left\{H_{1},\left(\frac{\partial H_{0}}{\partial \mathbf{v}_{\mathbf{0}}}\right)\right\}\right] \\
& -\left\{H_{0},\left(\frac{\partial H_{1}}{\partial \mathbf{v}_{1}}\right)\right\}^{T} \mathbf{D}_{\mathbf{1}}^{-1}\left[\left\{H_{0},\left(\frac{\partial H_{1}}{\partial \mathbf{v}_{\mathbf{1}}}\right)\right\}\right. \\
& \left.+u\left\{H_{1},\left(\frac{\partial H_{1}}{\partial \mathbf{v}_{\mathbf{1}}}\right)\right\}\right] \\
= & 0 \quad u
\end{aligned}
$$

Upon collecting terms that contain the singular control u, we get Eqs. (59) - (61).

q.e.d.

Remark 5 The above results can be obtained directly from those of the previous section by direct substitution for $\mathcal{H}_{0}$ and $\mathcal{H}_{1}$. Thus we can verify that

$$
\begin{aligned}
\mathcal{H}_{0} & =H_{0}\left(\mathbf{x}, \mathbf{p}, \mathbf{v}_{\mathbf{0}}\right) \\
\mathcal{H}_{1} & =H_{1}\left(\mathbf{x}, \mathbf{p}, \mathbf{v}_{\mathbf{1}}\right) \\
\left\{\mathcal{H}_{0}, \mathcal{H}_{1}\right\} & =\left\{H_{0}, H_{1}\right\}\left(\mathbf{x}, \mathbf{p}, \mathbf{v}_{\mathbf{0}}, \mathbf{v}_{\mathbf{1}}\right) \\
\left\{\mathcal{H}_{0},\left\{\mathcal{H}_{0}, \mathcal{H}_{1}\right\}\right\} & =\mathcal{A} \\
\left\{\mathcal{H}_{1},\left\{\mathcal{H}_{0}, \mathcal{H}_{1}\right\}\right\} & =\mathcal{B}
\end{aligned}
$$

Remark 6 Using the relation between the Poisson and the Lie Brackets, we can express all of the above results in terms of the Lie Brackets. Note in particular that

$$
\begin{aligned}
\left\{H_{0}, H_{1}\right\} & =\left\{\mathbf{p}^{T} \mathbf{f}, \mathbf{p}^{T} \mathbf{g}\right\}=\mathbf{p}^{T}(\mathbf{f}, \mathbf{g}) \\
\left\{H_{0},\left\{H_{0}, H_{1}\right\}\right\} & =\left\{\mathbf{p}^{T} \mathbf{f}, \mathbf{p}^{T}(\mathbf{f}, \mathbf{g})\right\}=\mathbf{p}^{T}(\mathbf{f},(\mathbf{f}, \mathbf{g})) \\
\left\{H_{0},\left(\frac{\partial H_{0}}{\partial \mathbf{v}_{0}}\right)\right\} & =\left\{\mathbf{p}^{T} \mathbf{f},\left(\frac{\partial \mathbf{f}}{\partial \mathbf{v}_{0}}\right)^{T} \mathbf{p}\right\} \\
& =\mathbf{p}^{T}\left(\mathbf{f}, \frac{\partial \mathbf{f}}{\partial \mathbf{v}_{0}}\right)
\end{aligned}
$$

where we define $\mathrm{p}^{T}(\mathbf{f}, \underset{v \mathbf{f}}{\sqrt{0}})$ as a $\left(m_{0} \times 1\right)$ column vector such that

$$
\mathbf{p}^{T}\left(\mathbf{f}, \frac{\partial \mathbf{f}}{\partial \mathbf{v}_{\mathbf{0}}}\right)_{i}=\mathbf{p}^{T}\left(\mathbf{f}, \frac{\partial \mathbf{f}}{\partial v_{0 i}}\right)
$$

And the other Poisson Brackets can be converted similarly. Thus,

$$
\mathcal{A}=\mathbf{p}^{T}(\mathbf{f},(\mathbf{f}, \mathbf{g}))
$$

$$
\begin{aligned}
+ & {\left[\mathbf{p}^{T}\left(\mathbf{g}, \frac{\partial \mathbf{f}}{\partial \mathbf{v}_{\mathbf{0}}}\right)\right]^{T}\left(\frac{\partial^{2} H_{0}}{\partial \mathbf{v}_{0}{ }^{2}}\right)^{-1}\left[\mathbf{p}^{T}\left(\mathbf{f}, \frac{\partial \mathbf{f}}{\partial \mathbf{v}_{0}}\right)\right] } \\
& -\left[\mathbf{p}^{T}\left(\mathbf{f}, \frac{\partial \mathbf{g}}{\partial \mathbf{v}_{\mathbf{1}}}\right)\right]^{T}\left(\frac{\partial^{2} H_{1}}{\partial \mathbf{v}_{\mathbf{1}}{ }^{2}}\right)^{-1}\left[\mathbf{p}^{T}\left(\mathbf{f}, \frac{\partial \mathbf{g}}{\partial \mathbf{v}_{\mathbf{1}}}\right)\right] \\
\mathcal{B}= & \mathbf{p}^{T}(\mathbf{g},(\mathbf{f}, \mathbf{g})) \\
& +\left[\mathbf{p}^{T}\left(\mathbf{g}, \frac{\partial \mathbf{f}}{\partial \mathbf{v}_{\mathbf{0}}}\right)\right]^{T}\left(\frac{\partial^{2} H_{0}}{\partial \mathbf{v}_{0}^{2}}\right)^{-1}\left[\mathbf{p}^{T}\left(\mathbf{g}, \frac{\partial \mathbf{f}}{\partial \mathbf{v}_{\mathbf{0}}}\right)\right] \\
& -\left[\mathbf{p}^{T}\left(\mathbf{f}, \frac{\partial \mathbf{g}}{\partial \mathbf{v}_{\mathbf{1}}}\right)\right]^{T}\left(\frac{\partial^{2} H_{1}}{\partial \mathbf{v}_{\mathbf{1}}{ }^{2}}\right)^{-1}\left[\mathbf{p}^{T}\left(\mathbf{g}, \frac{\partial \mathbf{g}}{\partial \mathbf{v}_{\mathbf{1}}}\right)\right]
\end{aligned}
$$

\section{Discussion}

From the expressions for $\mathcal{A}$ and $\mathcal{B}$ we note that the first terms are similar to the case of a single input. The only difference is that they are functions of $\mathbf{v}_{\mathbf{0}}$ and $\mathbf{v}_{\mathbf{1}}$ and we must substitute their optimal values in terms of $\boldsymbol{x}$ and $\mathbf{p}$ while evaluating those terms. The next two terms are due to the time derivatives of $\mathbf{v}_{\mathbf{0}}$ and $\mathbf{v}_{\mathbf{1}}$. Thus we can use the above formula even when $v_{0}$ and $\mathbf{v}_{\mathbf{1}}$ are constants or more general functions of $\mathbf{x}$ and $\mathrm{p}$ with the appropriate modifications. Even though the Lie Bracket solutions are given only for a first order singular arc, the Poisson Bracket solutions along with the Generalized Hamiltonians can be used for arcs of any order. Lemma 8 is also useful to obtain higher order derivatives recursively.

We have excluded the case where $\mathbf{f}$ and $\boldsymbol{g}$ are functions of time from our discussion. But by the standard procedure of introducing an extra state to represent the time, we can apply all our results to that case also.

We can also extend our results to the case of multiple linear controls occurring in the form

$$
\dot{\mathbf{x}}=\mathbf{f}\left(\mathbf{x}, \mathbf{v}_{\mathbf{0}}\right)+\sum_{i=1}^{\mathrm{a}} u_{i} \mathbf{g}_{\mathbf{i}}\left(\mathbf{x}, \mathbf{v}_{\mathbf{i}}\right)
$$

But there is a restriction on the product of the order of the singular arc corresponding to each $u_{i}$ and the number of $u_{i}$, in this case $\mathrm{s}$, since $\mathbf{x}$ and $\mathrm{p}$ can satisfy $2 n$ independent relations at the most.

We excluded bang-bang type (linear-saturating) and chattering type (non-linear, singular) controls from consideration due to the complex nature of the solution in those cases.

By a careful choice of the controls $\mathbf{v}_{\mathbf{0}}$ and $\mathbf{v}_{\mathbf{1}}$ (by using a transformation if necessary), one can make the Hessian matrices diagonal so that their inverses can be evaluated quite easily. 
Remark $\mathbf{7}$ For the special case where $\mathbf{v}_{\mathbf{1}} \equiv 0$, i.e., the only controls are $\mathrm{v}_{\mathrm{o}}$ and $\mathrm{u}$, the Legendre-Clebsch necessary condition for the problem considered in Eq.(40) reduces to

$$
\begin{aligned}
\mathcal{B}= & \mathbf{p}^{T}(\mathbf{g},(\mathbf{f}, \mathbf{g}))+ \\
& {\left[\mathbf{p}^{T}\left(\mathbf{g}, \frac{\partial \mathbf{f}}{\partial \mathbf{v}_{\mathbf{0}}}\right)\right]^{T}\left(\frac{\partial^{2} H_{0}}{\partial \mathbf{v}_{0}^{2}}\right)^{-1}\left[\mathbf{p}^{T}\left(\mathbf{g}, \frac{\partial \mathbf{f}}{\partial \mathbf{v}_{0}}\right)\right]>0 }
\end{aligned}
$$

Since we assumed that the Hessian matrix $\left(\frac{\partial^{2} H_{0}}{\partial \mathbf{v}_{0}{ }^{2}}\right)$ is negative definite, the second term in the above expression is always negative. Thus we have the following result.

Corollary $4 A$ necessary condition for the singular controlfor the above special case to be optimal is that

$$
\mathbf{p}^{T}(\mathrm{~g},(\mathbf{f}, \mathrm{g}))>0
$$

where we substitute the optimal value of $v_{0}$ an terms of $\mathbf{x}$ and $\mathbf{p}$ in the above expression.

\section{Example}

We illustrate the above results with the help of an example of optimal turn to a heading of a spacecraft in atmospheric flight at constant altitude. The equations of motion and the complete solution are given in Ref. (15) and we show that our results are identical to those given in that reference.

The equations of motion, in non-dimensional form, are given by

$$
\begin{aligned}
& \frac{d \xi}{d \theta}=u \cos \psi \\
& \frac{d \eta}{d \theta}=u \sin \psi \\
& \frac{d u}{d \theta}=\frac{\tau}{\mu}-\frac{u^{2}\left(1+\lambda^{2}\right)}{2 E^{*} \mu} \\
& \frac{d \psi}{d \theta}=\frac{u \lambda \sin \sigma}{\mu} \\
& \frac{d \mu}{d \theta}=-\tau
\end{aligned}
$$

where, $\xi$ and $\eta$ correspond to the $\mathrm{x}$ and y position coordinates, $u$ is the velocity, $\psi$ is the heading angle, $\mu$ is the mass and $\theta$ is the time in normalized form. The controls are $\lambda$, the lift coefficient, $\sigma$, the bank angle and I- the thrust. Since the flight is restricted to the horizontal plane, the flight-path angle is always zero and this gives a relation between the lift and the bank controls os

$$
\lambda \cos \sigma=\frac{\mu}{u^{2}}
$$

Selecting $\sigma$ as the independent control, eliminating $\lambda$ and using the transformation

$$
\alpha=\tan u
$$

the equations for $\mathrm{u}$ and $\psi$ can be re-written as

$$
\begin{aligned}
\frac{d u}{d \theta} & =\frac{\tau}{\mu}-\frac{u^{2}}{2 E^{*} \mu}\left(1+\frac{\mu^{2}}{u^{4}}\right)-\frac{\mu \alpha^{2}}{2 E^{*} u^{2}} \\
\frac{d \psi}{d \theta} & =\frac{\alpha}{\mathrm{U}}
\end{aligned}
$$

It is desired to maximize the final heading $\psi\left(t_{f}\right)$ for specified $\xi_{J}, \eta_{f}, \mathrm{u} J$ and $\boldsymbol{\mu}_{J}$.

Before we proceed, it is easy to recognize that $S,[$ and $\eta$ are ignorable. Thus, we have the following integrals of motion

$$
H=C_{0}, \quad p_{\xi}=C_{1} \text { and } p_{\eta}=C_{2}
$$

We can also verify, by direct differentiation and substitution that there exists one more integral

$$
p_{\psi}=C_{1} \eta-C_{2} \xi+C_{3}
$$

which is a result of the assumed spherical symmetry of the problem.

Since $\tau$ appears linearly, a singular arc is a possible sub-arc of the extremal. We would like to compute the value of I- along the singular arc using the results developed in the previous section. Comparing the equations of motion given above to Eq. (40), we see that $\mathbf{v}_{\mathbf{1}} \equiv 0$. Thus we have the special case discussed in Remark 8. Identifying the corresponding quantities, we get

$$
\left.\mathbf{f}=\left[\begin{array}{c}
u \cos \psi \\
u \sin \psi \\
a_{1}(u, \mu, \alpha) \\
\left(\frac{\alpha}{u}\right) \\
0
\end{array}\right] \quad \mathbf{g}=\quad \begin{array}{c}
0 \\
0 \\
\left(\frac{1}{u}\right) \\
-1
\end{array}\right]
$$

where,

$$
a_{1}=-\frac{u^{2}}{2 E^{*} \mu}\left(1+\frac{\mu^{2}}{u^{4}}\right)-\frac{\mu \alpha^{2}}{2 E^{*} u^{2}}
$$

The Lie Brackets can be calculated from the above as

$$
\begin{aligned}
& (\mathbf{f}, \mathbf{g})=\left[\begin{array}{c}
-\left(\frac{\cos \psi}{\mu}\right) \\
-\left(\frac{\sin \psi}{\mu}\right) \\
a_{2}(u, \mu, \alpha) \\
\left(\frac{\alpha}{\mu u^{2}}\right) \\
0
\end{array}\right] \\
& \left(\frac{\partial \mathbf{f}}{\partial \alpha}\right)=\left[\begin{array}{c}
0 \\
0 \\
-\left(\frac{\mu \alpha}{E^{\alpha} u^{2}}\right) \\
\left(\frac{1}{u}\right) \\
0
\end{array}\right]
\end{aligned}
$$




$$
\begin{aligned}
& \frac{\partial}{\partial \alpha}(\mathbf{f}, \mathbf{g})=-\left(\mathbf{g}, \frac{\partial \mathbf{f}}{\partial \alpha}\right) \\
& =\left[\begin{array}{c}
0 \\
0 \\
-\frac{\alpha}{E^{\circ} y^{2}}\left(\frac{2}{y}+1\right) \\
\left.\frac{1}{\mu u^{2}}\right) \\
0
\end{array}\right. \\
& \left(\mathbf{f}, \frac{\partial \mathbf{f}}{\partial \alpha}\right)=\left[\begin{array}{c}
\left(\frac{\mu \alpha}{E^{*} u^{2}}\right) \cos \psi+\sin \psi \\
\left(\frac{\mu \alpha}{E^{*} u^{2}}\right) \sin \psi-\cos \psi \\
-\left(\frac{2 \alpha}{E^{\alpha^{2} u}}\right) \\
\frac{1}{2 E^{*}}\left(\frac{1}{\mu}+\frac{\mu}{u^{4}}-\frac{\mu \alpha^{2}}{u^{4}}\right) \\
0
\end{array}\right] \\
& (\mathbf{g},(\mathbf{f}, \mathbf{g}))=\left[\begin{array}{c}
-\left(\frac{\cos \psi}{\mu^{2}}\right) \\
-\left(\frac{\sin \psi}{\mu^{2}}\right) \\
a_{3}(u, \mu, \alpha) \\
\frac{\alpha}{\mu^{2} u^{2}}\left(1-\frac{2}{u}\right) \\
0
\end{array}\right] \\
& (\mathbf{f},(\mathbf{f}, \mathbf{g}))=\left[\begin{array}{c}
a_{4}(u, \psi, \mu, \alpha) \\
a_{5}(u, \psi, \mu, \alpha) \\
a_{6}(u, \mu, \alpha) \\
a_{7}(u, \mu, \alpha) \\
0
\end{array}\right]
\end{aligned}
$$

where,

$$
\begin{aligned}
a_{2}= & \frac{1}{2 E^{* *}}\left(\frac{2 u}{\mu^{2}}-\frac{2}{u^{3}}+\frac{u^{2}}{\mu^{2}}-\frac{1}{u^{2}}\right) \\
& -\frac{\alpha^{2}}{E^{*}}\left(\frac{1}{u^{3}}+\frac{1}{2 u^{2}}\right) \\
a_{3}= & \frac{1}{\mu} \frac{d a_{2}}{d u}-\frac{d a_{2}}{d \mu} \\
a= & \frac{2 \alpha \sin \psi}{\mu u}-a_{2} \cos \psi \\
a_{5}= & -\frac{2 \alpha \cos \psi}{\mu u}-a_{2} \sin \psi \\
a_{6}= & \frac{d a_{2}}{\mathrm{du}} a_{1}-\frac{d a_{1}}{d u} a_{2} \\
a_{7}= & -\frac{2 \alpha}{\mu u^{3}} a_{1}+\frac{\alpha}{u^{2}} a_{2}
\end{aligned}
$$

We consider a special case where $\theta_{f}, \xi_{f}$ and $\eta_{f}$ are free. Then,

$$
C_{0}=C_{1}=C_{2}=0 \text { and } p_{\psi}=C_{3}
$$

We have the following integrals of motion

$$
H=p_{u} a_{1}+p_{\psi}\left(\frac{\alpha}{u}\right)=0
$$

$$
\begin{aligned}
\Phi & =p_{u}\left(\frac{1}{\mu}\right)-p_{\mu}=0 \\
\dot{\Phi} & =p_{u} a_{2}+p_{\psi}\left(\frac{\alpha}{\mu u^{2}}\right)=0 \\
\left(\frac{\partial H}{\partial \alpha}\right) & =-p_{u}\left(\frac{\mu \alpha}{E^{*} u^{2}}\right)+p_{\psi}\left(\frac{1}{u}\right)=0
\end{aligned}
$$

These are four equations in the four unknowns $\alpha, p_{u}, p_{\psi}$ and $p,$. Eliminating them, we get the switching curve in the $(u, \mu)$ space as

$$
\mu=\frac{u^{2}}{\sqrt{1+u}}
$$

and the control is given as

$$
a=\sqrt{2+u}
$$

Finally, the singular value of the thrust $\tau$ appears in the second derivative of the switching function $\Phi$ as

$$
\ddot{\Phi}=\mathcal{A}+\tau \mathcal{B}=0
$$

where, after using all the above relations and simplifying, we get,

$$
\begin{aligned}
\boldsymbol{A} & =-p_{\psi} \frac{\alpha}{E^{*} \mu^{2} u} \frac{(3 u+4)}{(1+u)} \\
\mathcal{B} & =p_{u} \frac{1}{E^{*} \mu^{3}} \frac{\left(4+5 u+2 u^{2}\right)}{(1+u)}
\end{aligned}
$$

Thus, eliminating $\alpha, p_{u}$ and $p_{\psi}$, we get

$$
\tau=-\frac{\boldsymbol{A}}{\mathcal{B}}=\frac{u^{2}(2+u)(4+3 u)}{E^{*}(1+u)\left(4+5 u+2 u^{2}\right)}
$$

All of the above results are identical to those obtained in Ref. (15), thus confirming the validity of our method.

\section{Conclusions}

The method presented here is an alternate way to obtain the singular control using the Lie and Poisson Brackets. The more direct method involves expressing all the non-singular optimal controls in terms of the state and the adjoint variables, substituting in the Hamiltonian and then finding the time derivatives of the switching function. This is a laborious process which often requires very clever algebraic manipulations. One more advantage of the present method is that it can be automated through symbolic manipulation. The contribution of the non-singular controls can be clearly identified through this solution and the coefficient of the singular control in the second derivative is explicitly given so that if one wants to check only the generalized Legendre-Clebsch condition, one can do so vary 
easily without evaluating the complete second derivative. There is scope for extending the method to more general cases, for example for problems with state and control equality constraints and we are currently working on that problem. Though Poisson Brackets, together with the Generalized Hamiltonian can be used in most cases for singular controls of any order, the solution can be expressed in terms of the original Hamiltonian and Lie Brackets only in some special cases as illustrated above.

\section{Appendix}

\section{Implicit Functions Theorem ${ }^{17}$}

Let $I ' \in R^{p}$ be a vector valued function defined on an open set $\mathrm{O} \in R^{p+q}$. Suppose I' $\mathrm{E} C^{1}$ on $\mathrm{O}$. Let $\left(\alpha_{0}, \beta_{0}\right)$ be a point in $\mathbf{O}$ for which $\Gamma\left(\alpha_{0}, \beta_{0}\right)=0$ and for which the $p \times p$ Jacobian $\left(\frac{\partial \Gamma}{\partial \alpha}\right)$ is non-singular. Then, there exists an open set $S \in R^{q}$ containing $\beta_{0}$ and one, and only one, vector valued function $\odot$ defined on $S$ and having values in $R^{p}$ such that

$$
\begin{aligned}
\Theta & \in C^{1} \text { on } S \\
\Theta\left(\beta_{0}\right) & =\alpha_{0} \\
\Gamma(\Theta(\beta), \beta) & =0 \forall \beta \in S
\end{aligned}
$$

and the partial derivative of $Q$ with respect to $\beta$ is given by

$$
\left(\frac{\partial \Theta}{\partial \beta}\right)=-\left(\frac{\partial \Gamma}{\partial \alpha}\right)^{-1}\left(\frac{\partial \Gamma}{\partial \beta}\right)
$$

\section{References}

[1] Speyer Jason L., "On the Fuel Optimality of Cruise," J. Aircraft, Vol. 10, No. 12, Dec. 1973, pp. 763-765.

[2] Schultz Robert L., "Fuel Optimality of Cruise," J. of Aircraft, Vol. 11, No. 9, Sept. 1974, pp. 586-587.

[3] Speyer Jason L., "Non-Optimality of Steady State Cruise for Aircraft," AIAA Journal, Vol. 14, No. 11, Nov. 1976, pp. 1604-1610.

[4] Bell D. J. and Jacobson D.H., Singular Optimal Control Problems, Academic Press, London, 1975.

[5] Kelley H. J., Kopp R. E., Moyer H.G., "Singular Extremals," Topics in Optimization, G.Lietmann ed., Academic Press, NY, 1967,pp. 63-101.

[6] Goh B.S., "The Second Variation for the Singular Bolza Problem," SIAM J. Control and Optimization, Vol. 4, 1966, pp. 309-325.
[7] Goh B.S., "Necessary Conditions for Singular Extremals Involving Multiple Control Variables," SIAM J. Control and Optimization, Vol. 4, 1966, pp. 716-731.

[8] Robbins H. M., "A Generalized Legendre-Clebsch Condition for the Singular Cases of Optimal Control," IBM J. of Research and Development, Vol. 11, 1967, pp. 361-372.

[9] Krener Arthur. J., "The High Order Maximal Principle And Its Application to Singular Extremals," SIAM J. of Control and Optimization, Vol.15, No.2, Feb. 1977, pp. 256-293.

[10] Gabasov R. and Kirillova F. M., "High Order Necessary Conditions for Optimality," SIAM J. of Control and Optimization, Vol.10, 1972, pp. 127-168.

[11] Bell D. J., "Lie Brackets and Singular Optimal Control," IMA J. of Mathematical Control and Information, Vol.1, 1984, pp. 83-94.

[12] G. Fraser-Andrews, "Finding Candidate Singular Optimal Controls : A State of the Art Survey," JOTA, Vol. 60, No.2, Feb. 1989, pp. 173-190.

[13] Lamnabhi-Lagarrigue F. and Stefani G., "Singular Optimal Control Problems : On the Necessary Conditions of Optimality," SIAM J. of Control and Optimization, Vol.28, No.4, July 1990, pp. 823-840.

[14] Arnold V. I., Mathematical Methods of Classical Mechanics, Springer-Verlag, New York, 1978.

[15] Nguyen. X. Vinh., Optimal Trajectories in Atmospheric Flight, Elsevier, New York 1981.

[16] Andrews Peter. B., An Introduction to Mathematical Logic and Type Theory : To Truth Through Proof, Academic Press, NY, 1986.

[17] Apostol Thomas S., Mathematical Analysis, Addison-Wesley, NY, 1974. 